\title{
DROUGHT ASSESSMENT OF IRAN USING THE MDI INDEX
}

\author{
Iman Kordpour ${ }^{\text {a }}$, Saeid Farzaneha ${ }^{\text {a }}$ Reza Shahhoseini ${ }^{\text {a* }}$ \\ ${ }^{a}$ School of Surveying and Geospatial Engineering, College of Engineering, University of Tehran, Tehran, Iran \\ (iman.kurdpour, farzaneh, rshahosseini)@ut.ac.ir
}

\author{
Commission VI, WG VI/4
}

KEY WORDS: GRACE, TWS, Drought, Perception, MDI

\begin{abstract}
:
Drought is one of the most common natural phenomena. Many indices using multiple data types have been created, and their success at recognizing periods of extreme wetness and dryness has been well documented. The merit of the method is the utilization of terrestrial water storage (TWS) variations from Gravity Recovery and Climate Experiment (GRACE) quantification of drought intensity. Alongside with these observations, we add precipitation data to equations. In this study, we analyze Merged-dataset Drought index (MDI) using GRACE-derived TWSA and precipitation in Iran, where most of the area is desert and mountain in the middle and South of the country. Our sample period is from January 2003 to December 2014. MDI shows a strong correlation with existing drought indices, especially with the Palmer Drought Severity Index (PDSI). Based on the obtained results, MDI indicates a moderate Drought event in 2008 and 2012-2015, which is compatible with the recorded result of PDSI. The longest drought took 22 months (from January 2008 to October 2009). Interestingly, the coefficient of correlation between MDI and PDSI is 0.67.
\end{abstract}

\section{INTRODUCTION}

Drought is one the most widespread natural phenomena, drought assessment is an important subject matter for water resources system and drought risk management ( $\mathrm{S}$. Quiring, 2007), which can prevent the proper management of drought detriment. Different drought indicators such as the Standardized Precipitation Index (SPI), Effective Drought Index (EDI) and Standardised Precipitation-Evapotranspiration Index (SPEI) have been developed for drought quantification and characterization using ground point data. One of the preliminary difficulties is the inconsistency of in-situ measurements available for drought monitoring; the observations are not available everywhere (i.e. Incompatible spatially), they do not have consecutive data records (i.e. Incompatible spatially), moreover, drought can be the result of water supply deficiencies in many different sources such as soil moisture, surface water, groundwater, evaporation, etc. measuring each of such details is dependant to a total water storage measurements which is difficult to do accurately. For this reason, we use the measurements from the Gravity Recovery and Climate Experiment (GRACE) mission. (M. Rodell, 2012)

The final products from GRACE can be explained as total water storage (TWS) anomalies. GRACE TWS anomalies contain groundwater, surface water, and soil moisture, as well as snowpack anomalies in appropriate areas. GRACE is capable of supplying measurements consistently in both space and time. It makes TWS measurements abling to be used as the dataset for drought monitoring (S. Bettadpur, 2012). Precipitation is most associated with drought. Deficiencies in precipitation cause agricultural or hydrological drought. This study uses in-site precipitation data. Iran Meteorological Organization (IMO), produces precipitation daily data for Iran and utilizes observation from 1950-present.

While GRACE TWS measure longer drought events, precipitation can have an instant impact on a region and changes in short-term scale. Precipitation is considered as an input to the hydrological cycle, while GRACE TWS measures the severity of water changes. Owing to this, a new quantitative index considering GRACE TWS and precipitation data can progress this assessment. The selected dataset correlates with two types of drought (meteorological drought and Hydrological drought), giving robustness to the MDI (M.J. Leblanc, 2009). Long et al. (2013) proposed using GRACE TWS measurements as an alternative to in-situ measurements for drought estimation in Texas and argued that TWS changes provide a more reliable indicator of water storage changes than disaggregated soil moisture and groundwater storage information (Long et al, 2013). Yirdaw et al. concluded that GRACE TWS is a reliable total water storage indicator that can be used for drought studies in the Canadian Prairie (Yerdaw et al, 2008). Chen et al. found GRACE TWS was useful in identifying drought in the Amazon River basin, and in particular, more accurately measured drought intensity as compared to climate and land-surface models that historically underestimate the intensity (Chen et al, 2009). Li et al. noted that GRACE TWS and NDVI correlate well, and both identify the same droughts, though GRACE TWS based droughts last longer than NDVI based droughts, partly due to vegetation senescence (Le et al, 2012). Li also noted that GRACE TWS was particularly valuable for its ability to give information below the surface (Le et al, 2012). While these studies found GRACE TWS to be a reliable drought indicator, none developed a method to quantitatively define drought based on GRACE TWS measurements. Previous studies described methods to integrate GRACE TWS measurements into Land Data Assimilation Systems, such as GLDAS, and use the resulting information to identify droughts, but this integration can be difficult and time-consuming. In the course of this research, Thomas et al. (2014) published a study describing a GRACE TWS-based quantitative method to measure the occurrence and severity of the hydrological

\footnotetext{
* Corresponding author
} 
drought. This study used GRACE TWS deficits to quantitatively identify drought onset, duration, and severity and matched GRACE TWS-identified events to known meteorological droughts (Thomas et al, 2014).

The remainder of this paper is organized as follows: section 2 (Data) expands on each dataset's relevance to the study and provides extensive background for each dataset being used. Section 3 discusses the creation of the Multidataset Drought Index (MDI), the development of the drought classification scheme. Section 4 discusses the nuances of the index and analyses selected drought events in detail. Section 5 concludes the study and makes future recommendations.

\begin{tabular}{|l|c|c|}
\hline Setting & \multicolumn{2}{|c|}{ A4 size paper } \\
\hline & Mm & inches \\
Top & 25 & 1.0 \\
Bottom & 25 & 1.0 \\
Left & 20 & 0.8 \\
Right & 20 & 0.8 \\
Column Width & 82 & 3.2 \\
Column Spacing & 6 & 0.25 \\
\hline
\end{tabular}

Table 1. Margin settings for A4 size paper

\section{DATA}

Each dataset used in this study was selected for its ability to identify water changes. GRACE TWS directly quantifies surface and subsurface water storage, and precipitation directly measures the incoming amount of rainfall and/or snow. GRACE TWS is the least mature dataset and is available beginning April 2002. Consequently, this study spans from January 2003 to December 2016.

\subsection{GRACE TWSA (Terrestrial Water Storage Anomaly)}

GRACE detects monthly changes in Earth's gravity field caused by mass redistribution, which, overland and after removal of the atmospheric contributions, are attributed primarily to the movement of water in various surface and subsurface hydrologic reservoirs (Wahr et al., 2004). GRACE provides Level-3 (Release 05) data that are global monthly $1.0^{\circ}$ gridded TWSA information from university of Texas Center for Space Research, which are demonstrated in centimeters of equivalent water thickness (Landerer and Swenson, 2012, S. Bettadpur, 2012). The data are available for the period from January 2003 to December 2016.

\subsection{Precipitation}

Precipitation datasets are some of the most important types of data used for drought and climate analysis. Precipitation data are collected from weather stations, weather radar, satellite and computer models. To make the data easier to use, many organizations derive products from the raw data. These derived products may start with station data, which estimate (or interpolate) the information between stations using different methodologies. Iran's daily precipitation data is obtained from the 135 synoptic stations of Iran Meteorological Organization (IMO). Afterward, Daily precipitation data is converted to monthly data. The observations are point-based and generate grid, using Inverse Distance Weighted (IDW) for interpolation.

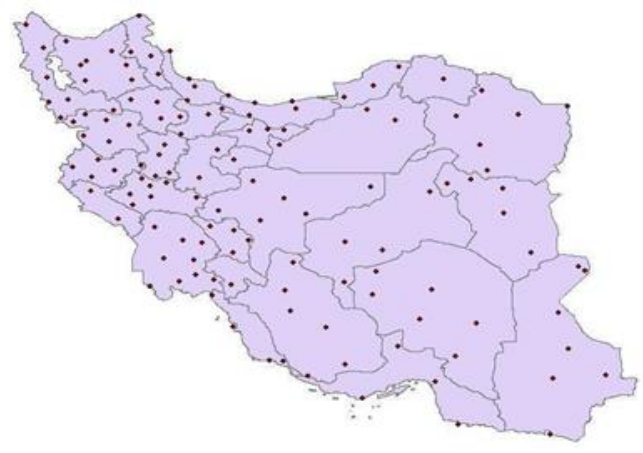

Figure 1. Precipitation stations of Iran

\subsection{Self-calibrated Palmer drought severity index (PDSI)}

The PDSI (Palmer 1965) can estimate the departure relative to normal situations in the surface water balance by using a hydrological accounting system (Dai et al. 2004; Heim 2002, W. M. Alley, 1984). The PDSI is primarily considered a meteorological drought indicator, and sometimes, an agricultural drought indicator (Wanders et al. 2010) Palmer (1965) formulated the PDSI with an objective to assess the variations in surface water balance. The PDSI integrates precursory and contemporary moisture reserve (precipitation $\mathrm{P}$ ) and demand (potential evapotranspiration (PE) into a hydrological accounting system, which includes a two-layer surface type model for soil moisture calculations. Central to the estimation of PDSI is the difference between the actual precipitation and the measure of precipitation needed to hold an appropriate soil moisture level for the same month. To improve spatial comparability, Wells et al. (2004) propounded a selfcalibrating PDSI (i.e., sc_PDSI) by replacing the empirically derived fixed values of the climatic characteristic (i.e., K) and the duration factors (0.897 and 1/3) used by Palmer (1965), based on data from the central United States, with values automatically calculated using historical climatic data of a specific region. Global grids $\left(2.5^{\circ} * 2.5^{\circ}\right)$ of monthly selfcalibrated PDSI data (http://www.esrl.noaa.gov/psd) are used here for the period from January 2003 to December 2016. The dataset utilized to compute self-calibrated PDSI and the methodology applied is elaborated in (Dai et al, 2004).

\subsection{Standardized Precipitation Evapotranspiration Index (SPEI)}

SPEI was investigating the effects of both precipitation and temperature on drought analysis. The SPEI is a multiscalar drought index like the SPI, based on climatic water balance (Vicente-Serrano et al. 2010a,b; Beguería et al. 2010). The SPEI has been calculated using the global 0.5 gridded ClimaticResearch Unit Time Series, version 3 (CRU TS3), monthly precipitation dataset (available at http://badc.nerc.ac.uk/browse/badc/cru/ data) and potential evapotranspiration estimated using Thornthwaite's method. Subsequently, a log-logistic distribution is fitted to determine SPEI values for the time period used. The SPEI dataset used here is procured from the Institutional Repository of the Spanish National Research Council (CSIC; https://digital.csic.es) covering the time period from January 2003 to December 2015. 


\begin{tabular}{|l|l|l|l|}
\hline Data & $\begin{array}{l}\text { Spatial } \\
\text { Resolutions }\end{array}$ & $\begin{array}{l}\text { Temporal } \\
\text { Resolutions }\end{array}$ & interval \\
\hline GRACE TWS & 1 degree & Monthly & $2003-2016$ \\
\hline Precipitation & point-based & Monthly & $2003-2016$ \\
\hline PDSI & 0.5 degree & Monthly & $2003-2016$ \\
\hline SPEI & 0.5 degree & Monthly & $2003-2015$ \\
\hline
\end{tabular}

Table 1. General description of the data

\section{PROPOSED METHOD}

A Z-score is a numerical measurement used in statistics of a value's relationship to the mean (average) of a group of values, measured in terms of standard deviations from the mean. Zscores reveal to statisticians and researchers whether a score is typical for a specified data set or if it is atypical. In addition to this, Z-scores also make it possible for analysts to adapt scores from various data sets to make scores that can be compared to one another accurately.

Before any analysis on the data, it must be standardized, especially when the data is multidimensional. The use of nonstandardized data may have an adverse effect on the results of the analyses. Data standardization helps ensure that their importance does not depend on the unit of measurement. As a result, standardized data is used in cases such as multivariate data analysis. Standardization is applicable to both quantitative and qualitative data.

In this study, GRACE TWS is used with precipitation data to produce a new drought index, known as Merged-dataset Drought Index (MDI). Both data correlate with two different types of drought. The data we choose has different spatial and temporal scales that must be conformable before estimating the index. Uniform spatial resolution is achieved by making a grid 1 degree in Iran. Uniform temporal resolution is achieved by creating a monthly time series for each dataset. Thereafter, we normalize Terrestrial water storage (TWS) and precipitation to estimate MDI.

$$
\begin{aligned}
& Z_{T W S=\frac{T W S(k j)-\overline{T W S}}{\sigma_{\text {TWS }}}} \\
& Z_{\text {precipitation }}=\frac{\text { Precipitation }(k)}{}-\overline{\text { Precrpitatron }} \\
& Z_{c}=Z_{\text {TWS }}+Z_{\text {Precipitation }} \\
& M D I=\frac{Z_{c}(K)-\overline{Z_{c}}}{\sigma_{Z_{e}}}
\end{aligned}
$$

We randomly select a month, for example, September. The MDI index is computed, as $Z_{T W S}$ denotes the standardized TWS in September; ( ${ }_{\text {Precipitation) denotes the standardized }}$ Precipitation in September; $\left(\sigma_{T W S}\right)$ denotes the standard deviation of TWS; ( $\left.\sigma_{\text {Precipitation }}\right)$ denotes the standard deviation of Precipitation. $\overline{\text { TWS }}$ Denotes the mean of TWS in September; $\overline{\text { Prectprtation }}$ denotes the mean of TWS in September; $\left(Z_{c}\right)$ denotes the mean of standardized TWS and Precipitation. $\left(\sigma_{Z_{i}}\right)$ denote the standard deviation of $Z_{e}$ MDI is calculated every month across regions beginning in January 2003. To compare MDI values spatially and temporally, a classification scheme is developed. The classes follow those currently used by the US Drought Monitor. This integrates interpretation of MDI into the current framework used for drought identification. (J.T. Carr, 1966)

\begin{tabular}{|l|l|l|}
\hline MDI value & Classification & Description \\
\hline 0 to -0.45 & Unclassified & Normal \\
\hline-0.45 to -0.90 & D0 & Abnormally Dry \\
\hline-0.90 to -1.35 & D1 & Moderate Drought \\
\hline-1.35 to -1.80 & D2 & Severe Drought \\
\hline-1.80 to -2.20 & D3 & Extreme Drought \\
\hline-2.20 and less & D4 & Exceptional Drought \\
\hline
\end{tabular}

Table 2. Drought classification based on MDI (Manacles, 2014)

Therefore, negative residuals are indicating deficits in land water storage compared to its mean, whereas positive residuals signify surplus water storage. Hence, in this study, drought events are identified by the persistence of negative WSDI values.

\section{RESULT}

Figure 1 reveals the comparison between time series of MDI and PDSI and SPEI index in Iran. To evaluate the comparison at inter-annual scales, a centered monthly moving is fitted to each of the time series. The lack of contemporaneity in the utilized datasets restricted the comparison to different time periods. So that the SPEI time series extended through the end of December 2015, respectively, PDSI and WSDI time series extended until the end of the study period.

The timescale for comparing MDI index and PDSI index is 14 years. Assess is between January 2003 to December 2016. In figure 1 , the time series of MDI and PDSI are represented by blue and red lines respectively. According to figure 1, the index MDI and PDSI show a descending trend.

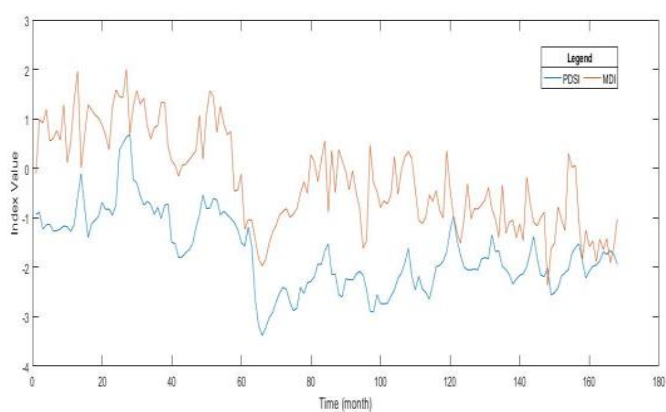

Figure 2. Relation between PDSI and MDI.

The observed behavior of the WSDI and its response to the climatic abnormally agrees reasonably well with the other indices explored. However, since WSDL is formulated using residual time series and satellite data, differences in treatment among the indices are envisaged. SPEI are more reactive to the rate of precipitation and evapotranspiration; as a result, higher magnitudes of fluctuations are observed in the time series of SPEI that consequently lead to poor correlation with WSDI. On the contrary, PDSI is rather incessant with relatively lower frequency variations, which are rather correlated with WSDI in evaluation to SPEI. The correlation coefficients show a fair correlation between PDSI and WSDI. However, it is important to note that in climatic zones (Iran) the value of PDSI is greater than that of WSDI, even though there is a high degree of compatibility in the timing of the drought events as represented by both the indices.

PDSI identifies droughts of 2006-2007 and 2008-2009 that show acute conditions compared to MDI. Computed values of 
the correlation coefficient $r$ between the MDI and the PDSI in Iran is 0.67. Treatment of MDI and PDSI is shown in figure 1.

\begin{tabular}{|c|c|c|c|}
\hline & MDI & PDSI & SPEI \\
\hline MDI & 1 & - & - \\
\hline PDSI & 0.67 & 1 & - \\
\hline SPEI & 0.43 & 0.39 & 1 \\
\hline
\end{tabular}

Table 3: Correlation matrix of drought indices computed for the Iran.

The inconsistencies noted in figure 1 between the drought indices are perhaps best explained by the basic differences in the type of data and method used in the calculation of the indices. The commonly used drought indices have some prominent constraints in their formulation. While SPEI is based on precipitation and evapotranspiration, Similarities noted in figure 1 between MDI and PDSI is the best explanation. In other words, the computation of PDSI involves a series of processes involving various water balance parameters. That is the reason why MDI and PDSI are significantly correlated.

\section{CONCLUSIONS}

In this study, data of TWSA gotten from GRACE satellites and Precipitation is used to calculate MDI as a robust drought index for large spatial scales. Each dataset was selected because it related to a different type of drought. GRACE TWS provides an integrated measure of water storage that considers surface and subsurface storage, which lends GRACE TWS to hydrological drought monitoring. Precipitation measures water input to an area. Precipitation events can have relatively immediate impacts and are useful for meteorological drought monitoring. Drought is estimated in 168 months (January 2003 to December 2015), and its trend and magnitude are studied. A monthly dataset is defined for each dataset for the period of consideration, and deviations from that normal are calculated. These deviations are transformed into z-scores used to calculate the Merged-dataset Drought Index. This computing method is not specific to a particular region, and it is also simple to Implementation and easy to understand. MDI is computed monthly for every region. MDI's strong correlation with current drought indices (such as PDSI) represents that it identifies droughts in a manner consistent with current practices. MDI is simpler to calculate than PDSI, and prepare the same information, making MDI a viable index for regions where PDSI is not available. A new drought classification scheme based on MDI is proposed. This scheme is created based on the regional Texas climate results, the similarity of the Texas climate to the Iran climate is the reason for using this classification. MDI successfully identified multiple droughts from 2003 - 2016 in every region of the country. Detected droughts generally happened in the same timespan across the regions, though the impact of the drought on the ecosystem varied. A drought event is identified when the MDI is negative. The most severe drought began in 2008-2009. We calculate the MDI index by integrating the data of TWS and precipitation. As a result, the Computed value of the correlation coefficient $r$ between the MDI, SPEI and the PDSI in Iran is 43 and 0.67 . 


\section{REFERENCES}

A.C. Thomas, J. Reager, J. Famiglietti, and M. Rodell. A GRACE-based water storage deficit approach for hydrological drought characterization. Geophysical Research Letters, 41:1537-1545, 2014.

Beguería, S., S. M. Vicente-Serrano, and M. Angulo-Martínez, 2010:A multiscalar global drought dataset: The SPEI base: A newgridded product for the analysis of drought variability and impacts.Bull.Amer. Meteor. Soc., 91, 1351-1356, doi:10.1175/2010BAMS2988.1.9.1

B. Li, M. Rodell, B. Zaitchik, R. Reichle, and R. Koster. Assimilation of GRACE terrestrial water storage into a land surface model: Evaluation 116 and potential value for drought monitoring in western and central Europe. NASA Publications, Paper 67, 2012.

Dai AG, Characteristics and trends in various forms of the palmer drought severity index during 1900- 2008. J Geophys Res-Atmos,

2011

116:D12115.https://doi.org/10.1029/2010jd015541

Dai A, Trenberth KE, Qian TT, A global dataset of palmer drought severity index for 1870-2002: relationship with soil moisture and effects of surface warming. Hydrometeorol 5:1117-1130, 2004. https://doi. org/10.1175/Jhm-386.1

Heim RR, A review of twentieth-century drought indices used in the United States. Bull Am Meteorol Soc 83:1149-1165 Wells N, Goddard S, Hayes MJ (2004) A self-calibrating palmer drought severity index. J Clim 17:2335-2351, 2002. https://doi.org/10.1175/15200442(2004)017<2335:ASPDSI>2. $0 . \mathrm{Co} ; 2$

J.T. Carr. Texas Droughts: Causes, Classification and Predication. Technical Report 30, Texas Water Development Board, 1966.

Landerer, F. W., and S. C. Swenson, Accuracy of scaled GRACE terrestrial water storage estimates. Water Resour. Res., 48, 2012, W04531, doi:10.1029/2011WR011453.

Vicente-Serrano, S., M. S. Beguería, and J. I. López-Moreno, A multiscalar drought index sensitive to global warming: The standardized precipitation evapotranspiration index. J. Climate, 23, 1696-1718, 2010a, doi:10.1175/2009JCLI290

D. Long, B. Scanlon, L. Longuevergne, A. Sun, D. Fernando, and H. Save. GRACE satellite monitoring of large depletion in water storage in response to the 2011 drought in Texas. Geophysical Research Letters, 41:3395-3401, 2013

J.L. Chen, C. Wilson, B. Tapley, Z. Yang, and G. Niu. 2005 drought event in the Amazon River basin as measured by GRACE and estimated by climate models. Journal of Geophysical Research-Solid Earth, 2009.
M. Rodell. Satellite Gravimetry Applied to Drought Monitoring, Remote Sensing of Drought Innovative Monitoring Approaches, pages 261-278. CRC Press, 2012.

M.J. Leblanc, P. Tregoning, G. Ramillien, S. Tweed, and A. Fakes. Basinscale, integrated observations of the early 21st century multiyear drought in southeast Australia. Water Resources Research, 2009.

S. Bettadpur. UTCSR Level-2 Processing Standards Document. Technical report, Center for Space Research, The University of Texas at Austin, 2012.

S. Quiring, J. W. Nielsen-Gammon, R. Srinivasan, T. Miller, and B. Narasimhan. Drought Monitoring Index for Texas. Technical report, Texas Water Development Board, 2007

S.Z. Yirdaw, K.R. Snelgrove, and C.O. Agboma. GRACE satellite observations of terrestrial moisture changes for drought characterization in the Canadian Prairie. Journal of Hydrology, $365: 84-92,2008$.

W. M. Alley. The Palmer Drought Severity Index: limitations and assumptions. Journal of Climate and Applied Meteorology, 23:1100-1109, 1984.

Wahr, J., S. Swenson, V. Zlotnicki, and I. Velicogna, Timevariable gravity from GRACE: First results. Geophys. Res Lett., 31, 2004. L11501, doi:10.1029/2004GL019779.

Wanders N, Lanen HAJv, Loon AFv, Indicators for drought characterization on a global scale. Wageningen University: Wageningen, Netherlands, 2010. Available online: http://www.eu-watch.org/publications/technical-reports/3

W.C. Palmer. Meteorological Drought. Technical Report 45, U.S.Weather Bureau, 1965. 